\title{
A serious fit of cough: A 66-year-old patient with myasthenia gravis presenting with spontaneous intercostal lung herniation after coughing. Case report and pathophysiological discussion
}

\author{
Riccardo Gerloni, ${ }^{1}$ Nicola Artusi, ${ }^{1}$ Ugo Giulio Sisto, ${ }^{1}$ Saverio Tollot, ${ }^{2}$ Roberto Copetti ${ }^{1,3}$ \\ ${ }^{1}$ Emergency Department, University Hospital of Cattinara; ${ }^{2}$ Radiology Department, University Hospital of \\ Cattinara, Trieste; ${ }^{3}$ Emergency Department, Hospital of Latisana, Latisana, Italy
}

\begin{abstract}
We present a case of a 66-year-old man with history of myasthenia gravis, severe obesity and osteopenia self-presented to our Emergency Department (ED) with severe pain in his left hemithorax, occurred after an episode of cough three days before. No history of trauma was reported. The patient underwent a chest-XR showing uncomplicated spontaneous fractures of the $5^{\text {th }}$ and $6^{\text {th }}$ left ribs. He was therefore discharged with appropriate analgesic treatment. Five days later, the patient came back to our ED for a wide left abdominal hematoma, though hemodynamically stable and eupneic. A CT-scan with contrast showed a rare and unexpected spontaneous left intercostal lung herniation complicated with a diffuse subcutaneous emphysema, pneumothorax, loculated bilateral pleural effusion and abdominal hematoma. The patient was admitted, treated conservatively and safely discharged after two weeks. We also provide a pathophysiological discussion of the case and a literature review.
\end{abstract}

\section{Case presentation}

A 66-year-old man self-presented to our Emergency Department (ED) with severe and increasing pain in his left hemithorax, occurred after an episode of cough three days before. No history of trauma was reported. The patient had medical history

\author{
Correspondence: Nicola Artusi, Medicina d'urgenza, Strada di Fiume \\ 447, 34149 Trieste TS, Italy \\ E-mail: nicola.artusi.1@gmail.com \\ Key words: Lung herniation; spontaneous; pulmonary hernia; obesity; \\ myasthenia gravis. \\ Conflict of interest: No one. This work was not supported by any grant. \\ Ethics approval and consent to participate: The manuscript does not \\ contain any elements that would allow the recognition of the patient. \\ Received for publication: 21 February 2020. \\ Accepted for publication: 18 May 2020. \\ This work is licensed under a Creative Commons Attribution 4.0 \\ License (by-nc 4.0). \\ CCopyright: the Author(s), 2020 \\ Licensee PAGEPress, Italy \\ Emergency Care Journal 2020; 16:8911 \\ doi:10.4081/ecj.2020.8911
}

of myasthenia gravis, severe obesity, ulcerative colitis and type-2diabetes in chronic treatment with prednisone $12,5 \mathrm{mg} / \mathrm{day}$, repaglinide and metformin. In 2006, he was diagnosed with osteopenia and a spontaneous vertebral compression fracture. The patient was also an active smoker.

He underwent a chest-RX (CXR) which demonstrated compound fractures of the $5^{\text {th }}$ and $6^{\text {th }}$ left ribs (Figure 1), classified as uncomplicated and spontaneous. The patient was therefore discharged with appropriate analgesic treatment.

Five days later, the patient came back to our ED for a wide left abdominal hematoma. The patient was hemodynamically stable and eupneic. The only symptom he reported was some tenderness in the site of the rib fractures occurred on the same day. He denied once again any traumas. The physical examination was inconclusive: a wide hematoma was found in the left side of the abdomen; the left rib cage was tender at superficial palpation and the respiratory sounds were reduced in the inferior left lung field.

Ordinary laboratory tests were taken, showing normal hemoglobin levels, leukocytosis (WBC: $18,2 \times 10^{3} \mu \mathrm{L}$ ), increased Creactive protein (CRP: $281,3 \mathrm{mg} / \mathrm{L}$ ) and procalcitonin (PCT: 0,34 $\mathrm{ng} / \mathrm{mL}$, normal value $<0,05$ ). The Arterial Blood Gas (A.B.G.) demonstrated a moderate respiratory insufficiency with chronic compensated respiratory acidosis ( $\mathrm{pH} 7,4$; $\mathrm{pCO} 2$ 48,4 mmHg; $\mathrm{pO}_{2}$ $\left.89 \mathrm{mmHg} ; \mathrm{HCO}_{3}-29 ; \mathrm{PaO}_{2} / \mathrm{FiO}_{2}=287\right)$. Coagulation and other blood tests were all in the normal range normal.

A plain CXR was performed, showing a displacement of the rib fragments, possible bibasal consolidations and a left pleural effusion (Figure 2). The lung ultrasound pattern was unclear, showing a diffuse ultrasonographic barrier effect.

In the suspicion of a hemothorax, a complete chest and abdominal CT-scans with contrast were performed, describing an unexpected picture of moderate intercostal lung herniation $(2,3 \mathrm{~cm})$ in the 5th space, a diffuse subcutaneous emphysema from the neck to the abdomen, a minimal left-sided pneumothorax and mild-tomoderate bilateral pleural effusion, partially loculated in the left side. The CT-scan also confirmed a non-refurnished abdominal wall hematoma and the known $5^{\text {th }}$ and $6^{\text {th }}$ ribs detached fractures (Figures 3-5).

Our patient underwent an urgent surgical consult, suggesting a conservative approach in a medical ward. The patient did not require non-invasive ventilatory supports, but we administered oxygen via face mask and nasal canulae. A prophylactic antibiotic regimen was started first with amoxicillin/clavulanic acid then switched to piperacillin/tazobactam on the 4th day. During the hospitalization, $\mathrm{Hb}$ values decreased to a minimum of $12,0 \mathrm{~g} / \mathrm{dL}$. No blood transfusions were prescribed. The patient was discharged after 15 days, with complete normalization of the clinical picture. Further CXRs in the following months described a complete spontaneous reduction of the hernia. 


\section{Discussion}

Spontaneous rib fractures are a common complication in osteopenic patients affected by respiratory infections, particularly in those patients treated chronically with corticosteroids. The initial evaluation and management of spontaneous stress fractures of the ribs involve the ruling out of main complications such as pneumothorax and thoracic bleeding. Further, a correct pain management is required: an association of paracetamol, NSAIDs/COX-2 inhibitors and as needed opioids (i.e. codeine, tramadol, etc.) is suggested, with an increasing role of ultrasound-guided locore- gional anesthesia. ${ }^{1}$ Since the surgical fixation is rarely needed, the usual approach considers early discharge and conservative therapy as first choice.

The late development of a thoracic and abdominal wall hematoma suggested a delayed intercostal hemorrhage. ${ }^{2}$ The patient never reported the typical signs or symptoms of intercostal lung herniation such as a palpable soft mass or worsening dyspnea. Though a thoracic wall hematoma is frequently associated with lung hernias, it's unspecific for this condition. ${ }^{3}$ The initial physical examination was possibly misled by the severe obesity of the patient. Despite the attested sensitivity and specificity of chest ultrasound in the ED for pleural effusions, rib fractures and pneu-
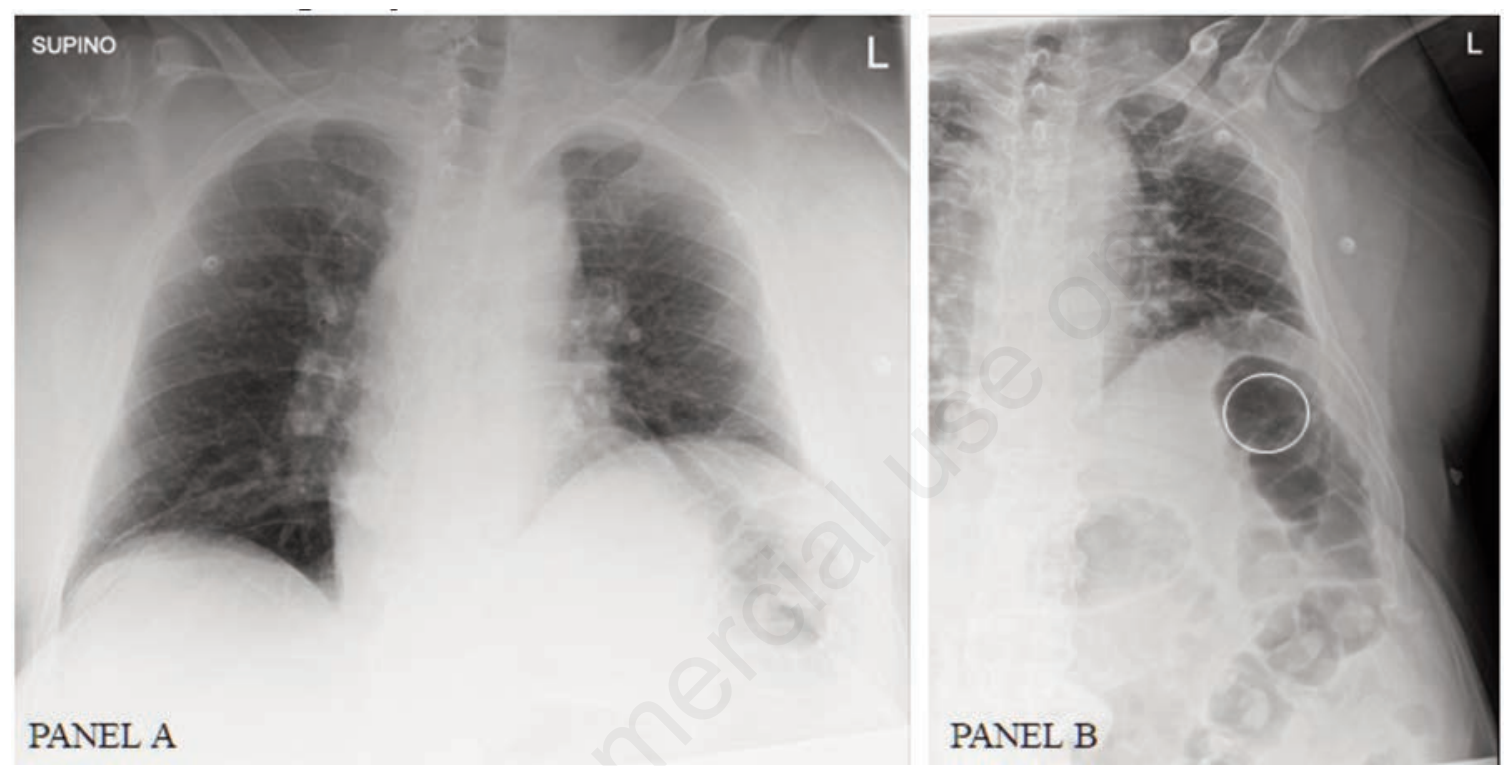

Figure 1. laying AP CXR (panel A) and left hemithorax (panel B) showing a compound antero-lateral fracture of the $5^{\text {th }}$ and $6^{\text {th }}$ left ribs (in the circle) and an elevation of the left hemidiaphragm.

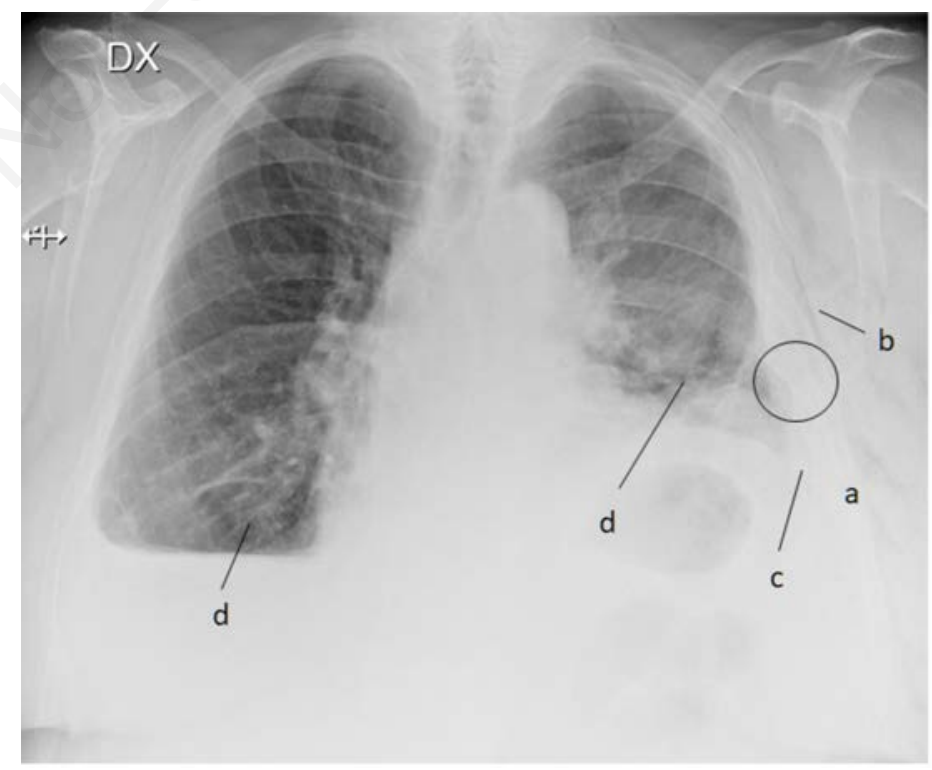

Figure 2. Standing AP CXR showing possible detachment of the fracture lines (a), subcutaneous emphysema (b), left pleural effusion (c), possible bibasal consolidations and a prominent elevation of the left hemidiaphragm (d). 
mothorax and, therefore, it's possible role in the detection of a lung herniation, ${ }^{4}$ the concomitant presence of obesity and subcutaneous emphysema reduced the diagnostic power of lung ultrasound. ${ }^{5}$ The first standing CXR was inconclusive. A CT-scan with contrast was therefore indicated in this condition. ${ }^{6}$ The CT-scan with contrast showed a self-limiting intercostal hemorrhage with no active bleeding, not needing for interventional hemostasis or blood transfusions. The CT-scan interestingly reported a lung herniation and subcutaneous emphysema, too. Lung herniations are rare findings in non-traumatic fractures, mostly associated with corticosteroid chronic therapies, primitive pulmonary affections, or previous invasive procedures. ${ }^{10,11}$ In our patient, the predisposing factor is likely to be the prednisone therapy. Corticosteroid-induced osteopenia is a common side-effect in chronic corticosteroid treatments; (in these patients, the bone samples show a lower density in osteoblasts and an increased rate of osteocyte apoptosis, with a general reduction in bone strength. On the other hand, glucocorticoid effects include an increase in osteoclasts lifespan). ${ }^{12-15}$

Anyway, the rib fractures alone can't explain the lung herniation, thus a global thoracic wall weakness needed to be present.
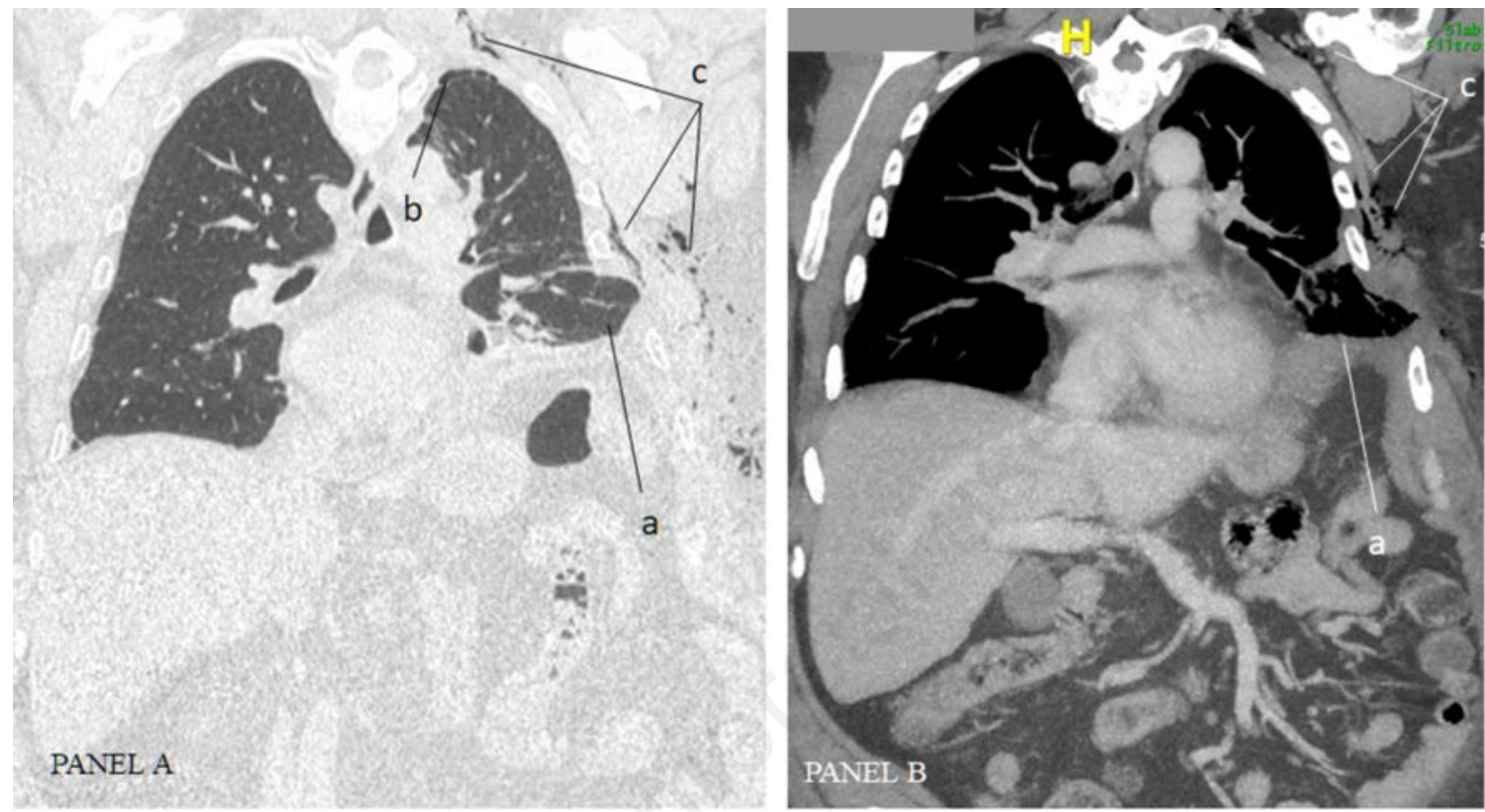

Figure 3. CT-scan coronal sections in lung window (panel A) and soft tissue window (panel B) showing the pulmonary herniation (a), the minimal pneumothorax (b) and the diffuse subcutaneous emphysema (c).
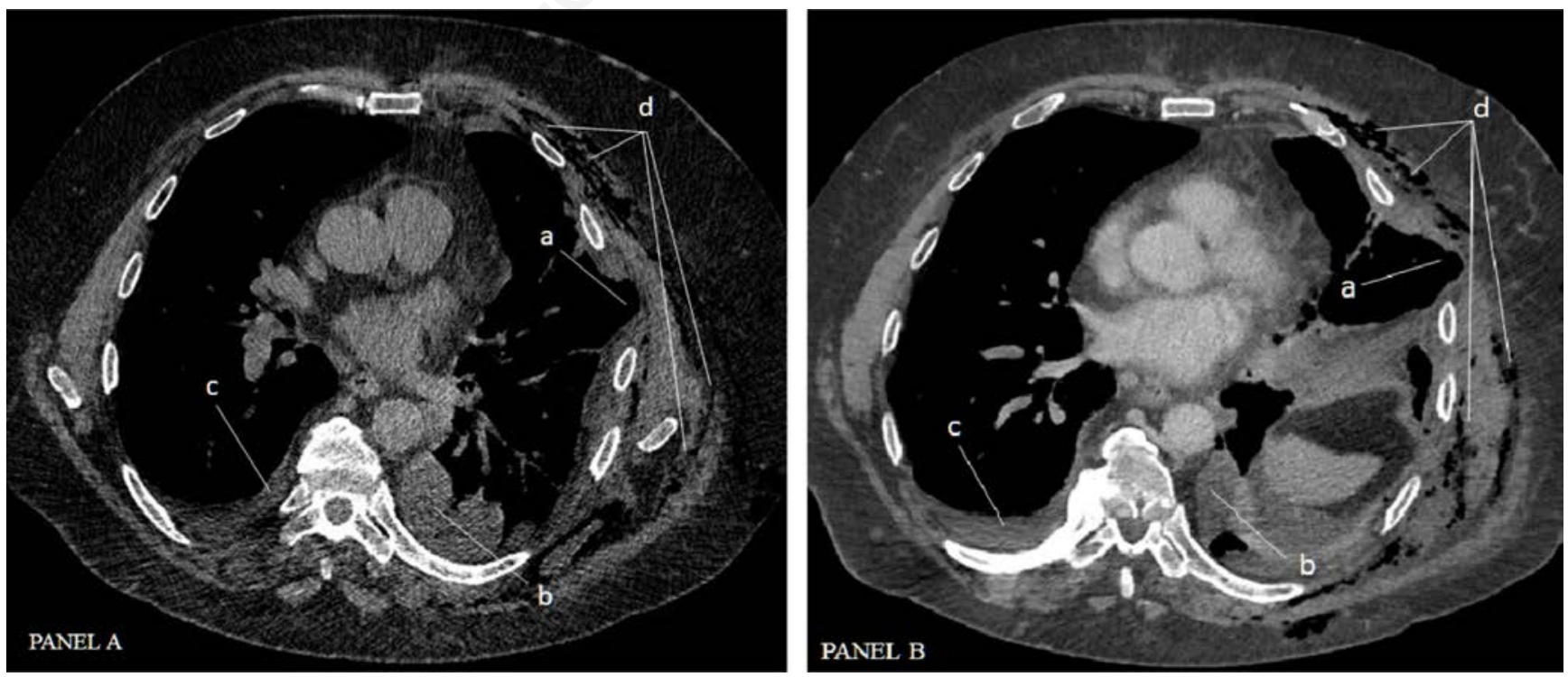

Figure 4. CT-scan transverse sections (cranial, panel A, and caudal including the spleen in panel B) showing the pulmonary herniation (a), the left sided loculated pleural effusion (b), the right sided pleural effusion (c) and the diffuse subcutaneous emphysema (d). 

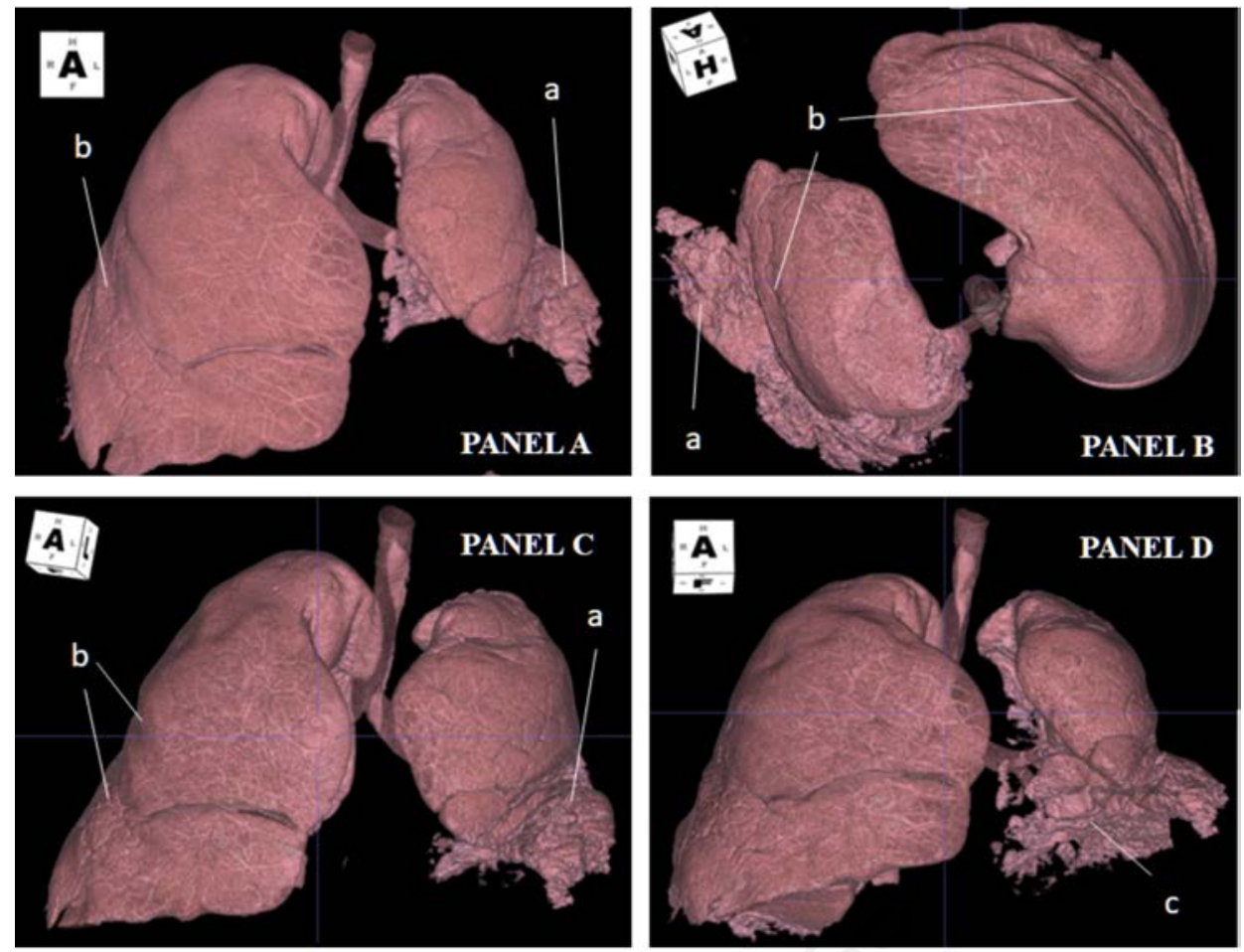

Figure 5. CT-scan, 3D-volume rendered reconstruction of the lungs, showing a) the left herniation, b) the rib footprints on the lung parenchyma, c) the parenchymal compression secondary to the pleural effusion in right lateral-anterior (panel A), superior (panel B) left lateral-anterior (panel C) and antero-inferior (panel D) views.

Actually, corticosteroid activity over muscle catabolism is well known: glucocorticoids inhibit protein synthesis while stimulating protein degradation, possibly leading to a skeletal muscle atrophy in several acute and chronic condition. Glucocorticoid-induced myopathy is a common complication in Cushing-like-syndromes which induce diffuse atrophy and substitution of muscle fibres, affecting both limbs and respiratory muscles. ${ }^{16,17}$

Severe obesity can be involved in the pathogenesis of lung herniation, too, both due to its increasing effect on intrathoracic pressure, and to a diffuse respiratory muscle overstretching and related weakness. ${ }^{18}$

Myasthenia Gravis (MG) affects the neuromuscular junction, resulting in a localized or diffuse muscle weakness which can involve the respiratory system. Many clinical conditions can exacerbate MG, as infections. Even if the idea of MG possibly involved in the pathogenesis of lung herniation is appealing, our patient didn't show any sign of myasthenic crisis as dyspnoea, fatigue or severe respiratory insufficiency; thus, a direct involvement of MG is unlikely. ${ }^{19,20}$ The patient was treated conservatively. Though some authors suggest an early interventional approach, ${ }^{21}$ in minor hernias, a watch-and-see conservative regimen, based on pain management and rest, could be sufficient to obtain a complete spontaneous resolution. Admission to hospital in case of acute complications is suggested. In case of large hernias or severe complications, as hernial incarceration or strangulation, with lung necrosis, a surgical reduction is required both with invasive or thoracoschopic approaches. ${ }^{22}$ Surgery is also the treatment of choice in combined lung and intestinal hernias and lung hernias involving the diaphragm. Our patient presented a moderate intercostal herniation with a risk for acute medical complications and therefore an in-hospital medical treatment was needed, with no surgical indications. $^{23}$

A clinical diagnosis of pneumonia could be debated. WBC count, CRP and PCT were mildly increased, suggesting a possible bacterial infection ${ }^{24,25}$ The patient never developed fever, and the serial hemocultures were constantly negative. The finding of pleural effusion, absent in the first hospital presentation while moderate at the second admission, could be consistent either with infection or with an inflammatory response. PCT was later negative after one week of antibiotic therapy.

\section{Conclusions}

The lung herniation is a rare either congenital or acquired condition, mostly following thoracic surgical procedures or thoracic traumas. Few cases of spontaneous lung herniation are reported in literature, most of them associated with rib fractures and persistent coughing, mechanical ventilation with positive pressure, emphysema and COPD. The surgical reduction of the hernia is the usual therapeutic option in these reported cases.

Our case depicts a spontaneous lung herniation associated with pneumothorax and subcutaneous emphysema after atraumatic rib bone fractures in a patient in chronic corticosteroid therapy for his history of myasthenia gravis. We successfully treated this patient with a conservative approach. The pathophysiological mechanism of the lung herniation in this patient is due to the side effects of a long-term glucocorticoid therapy on the thoracic wall, muscles and bones. MG is unlikely to be a causal factor. 


\section{References}

1. Karmakar M, Ho AM-H. Acute pain management of patients with multiple fractured ribs. J Trauma 2003;54:615-25.

2. Talbot BS, Gange CP Jr, Chaturvedi A, et al. Traumatic Rib Injury: Patterns, Imaging Pitfalls, Complications, and Treatment. Erratum In: Radiographics 2017;37:1004.. Radiographics 2017;37:628-51.

3. Maeda T, Sato R, Luthe SK, Russell MC. Spontaneous Intercostal Lung Hernia. Am J Med 2017;130:e399-e400.

4. O' Mahony AM, Murphy KM, O'Connor TM, Curran DR. Spontaneous pulmonary hernia secondary to intercostal muscle tear. BMJ Case Rep 2019;12:e231706.

5. Lee FCY. The Curtain Sign in Lung Ultrasound. J Med Ultrasound 2017;25:101-4.

6. Hallifax RJ, Talwar A, Wrightson JM, Edey A, Gleeson FV. State-of-the-art: Radiological investigation of pleural disease. Respir Med 2017;124:88-99. vol. 124.

7. Cox M, Thota D, Trevino R. Spontaneous Lung Herniation Through the Chest Wall. Mil Med 2018;183:e233-4.

8. Gaude GS, Chatterji R, Bagga AS, Naik AG, Bellad V. Spontaneous lung hernia. Indian J Chest Dis Allied Sci 1997;39:65-8.

9. Ross RT, Burnett CM. Atraumatic lung hernia. Ann Thorac Surg 1999;67:1496-7.

10. Haneda H, Okuda K, Nakanishi R. Case of intercostal lung hernia with hemosputum that developed after thoracoscopic lobectomy. Asian J Endosc Surg 2019;12:449-51.

11. Haro A, Komiya K, Taguchi Y, et al. A rare case of an intercostal lung herniation with fractures of the fifth and sixth ribs after thoracic surgery. Int J Surg Case Rep 2017;41:473-6.

12. Hofbauer LC, Gori F, Riggs BL, et al. Stimulation of Osteoprotegerin Ligand and Inhibition of Osteoprotegerin Production by Glucocorticoids in Human Osteoblastic Lineage Cells: Potential Paracrine Mechanisms of GlucocorticoidInduced Osteoporosis. Endocrinol 1999;140:4382-9.

13. Khosla S. Minireview: The OPG/RANKL/RANK System. Endocrinol 2001;142:5050-5.
14. Weinstein RS, Jilka RL, Michael Parfitt A, Manolagas SC. Inhibition of osteoblastogenesis and promotion of apoptosis of osteoblasts end osteocytes by glucocorticoids potential mechanisms of their deleterious effects on bone. J Clin Invest 1998;102:274-82.

15. Weinstein RS. Clinical practice. Glucocorticoid-induced bone disease. N Engl J Med 2011;365:62-70.

16. Yang T, Li Z, Jiang L, Xi X. Corticosteroid use and intensive care unit-acquired weakness: a systematic review and metaanalysis. Crit Care 2018;22:187.

17. GuptaY, Gupta A. Glucocorticoid-induced myopathy: Pathophysiology, diagnosis, and treatment. Indian J Endocrinol Metab 2013;17:913.

18. Behazin N, Jones SB, Cohen RI, Loring SH. Respiratory restriction and elevated pleural and esophageal pressures in morbid obesity. J Appl Physiol 2010;108:212-8.

19. Gilhus NE, Verschuuren JJ. Myasthenia gravis: Subgroup classification and therapeutic strategie. Lancet Neurol 2015;14:1023-36.

20. D. Drachman D. Myasthenia Gravis. Semin Neurol 2016;36:419-24.

21. Berry B, Ghazaleh D, Matar R, et al., Acquired intercostal lung herniation: conservative management may lead to continuation of symptoms and other adverse consequence. Gen Thorac Cardiovasc Surg 2020;68:403-7.

22. Batıhan G, Yaldız D, Ceylan KC. A rare complication of videoassisted thoracoscopic surgery: lung herniation retrospective case series of three patients and review of the literature. Wideochir Inne Tech Maloinwazyjne 2020;15:215-9.

23. François B, Desachy A, Cornu E, et al. Traumatic pulmonary hernia: surgical versus conservative management. J Trauma 1998;44:217-9.

24. Wussler D, Kozhuharov N, Tavares Oliveira M, et al. Clinical Utility of Procalcitonin in the Diagnosis of Pneumonia. Clin Chem 2019;65:1532-42.

25. Çolak A, Yllmaz C, Toprak B, Aktoğu S. Procalcitonin and CRP as Biomarkers in Discrimination of CommunityAcquired Pneumonia and Exacerbation of COPD. J Med Biochem 2017;36:122-6. 